\title{
Estilos atributivos en universitarios: comparaciones por género
}

\author{
Ana C. Díaz (1), Susana I. Aguirre (2), Carolina Jiménez (2) y Perla J. Jurado (2)* \\ (1) OPD Hospital Civil de Guadalajara, Universidad de Guadalajara, Guadalajara, Jalisco, México. \\ (2) Universidad Autónoma de Chihuahua. Facultad de Ciencias de la Cultura Física. DES Salud CAs 121 y 135. \\ Chihuahua, Chihuahua, México (correo-e: anacitlalli@gmail.com, siaguirre@uach.mx, cajimenez@uach.mx, \\ pjurado@uach.mx).
}

* Autor a quien debe ser dirigida la correspondencia.

Recibido Nov. 29, 2019; Aceptado Ene. 27, 2020; Versión final Mar. 6, 2020, Publicado Ago. 2020

\section{Resumen}

En este estudio se comparan los perfiles de estilos atributivos en el área de logros académicos en universitarios mexicanos. La muestra total fue de 1285 participantes; 660 mujeres y 625 hombres, con una edad media de 20.51 y 20.83 años respectivamente. La investigación fue de enfoque cuantitativa, descriptiva, tipo encuesta. Los participantes contestaron la versión informatizada del Cuestionario de Estilos Atributivos en el área de logros académicos adaptado previamente por los autores el año 2016. Los análisis de varianza multivariante y univariados, muestran que las mujeres en comparación con los hombres reportan mayores niveles de control interno en las dimensiones atribución a la suerte, atribución a la habilidad, atribución al profesor y sin diferencias en atribución al esfuerzo. Se concluye que dichas diferencias sugieren que al diseñar cualquier intervención para promover el cambio en los estudiantes hacia un estilo de control más interno habrá que tomar en cuenta al género.

\section{Attributional styles in university students: comparisons by gender}

\begin{abstract}
This study compares the profiles of attributive styles in the area of academic achievements among Mexican university students. The sample consists of 1285 participants including 660 women and 625 men, with an average age of 20.51 years and 20.83 years respectively. A quantitative approach with a descriptive and transversal survey design was used. All the participants completed the computerized version of the Attributive Styles Questionnaire (adapted in 2016) in the area of academic achievement. The one-way multivariate and univariate analysis of variance showed that women report higher levels of internal control than men in three of the dimensions: attribution to luck, attribution to ability, and attribution to the professor. There were no differences found between genders in attribution to effort. In conclusion, the data suggest that the gender variable should be included when designing any type of intervention that aims to promoting change towards a more internal style of control in students.
\end{abstract}

Keywords: attributional styles; student's beliefs; gender differences; self-perception 


\section{INTRODUCCIÓN}

Al hablar de atribución se hace referencia al proceso de asignación de causas del comportamiento propio o el de las otras personas; esta atribución puede ser interna llamada atribución personal o disposicional; o externa conocida como situacional (Mkumbo y Amani, 2012). El ser humano como tal, siempre está en constante búsqueda de dichas causas y sus porqués, de ahí la importancia del estudio de los estilos de atribución. En la literatura científica se encuentran diversas definiciones del término estilos de atribución o estilo atribucional; además es muy frecuente encontrar que se hable de locus de control o estilos de atribución indistintamente, en su revisión Visdómine-Lozano y Luciano (2006) aseveran que la atribución y el locus de control son equivalentes. la mayoría de los autores coinciden en definirlos como la manera en que las personas explican las causas de los eventos tanto negativos como positivos y las interpretaciones que les dan a éstos. Estas atribuciones pueden ser internas llamadas también atribución personal, disposicional o locus de control interno y las atribuciones externas también conocidas como situacionales o locus de control externo (O'Donnell et al., 2013; Peterson, 1991; Rotter, 1966; Salvador y Mayoral, 2011; Tavakolizadeh y Ebrahimi, 2011; Weiner, 2014). El locus de control externo se refiere a factores que no dependen del sujeto, como la creencia en elementos tales como una estrella, la suerte, la fortuna, etc. Mientras que, el comportamiento interno trae una relación entre su acción y el resultado, el locus externo tiene un nexo directo entre su acción y los factores externos. Un aspecto importante del comportamiento es el locus de control, formulado en el marco de la teoría del aprendizaje social; relacionado con la medida en que un individuo está motivado, dirigido o controlado, es decir, su marco de referencia interno, y la medida en que el entorno, como la suerte, la oportunidad, etc., influye en su comportamiento. El locus de control tiene que ver con la asignación de responsabilidad por el resultado de eventos o comportamientos; donde, la aparición de eventos a veces agradables o poco placenteros, alentadores, gratificantes o desconcertantes pueden denominarse refuerzos y las personas los perciben de manera diferente, lo que se caracteriza por un control interno o externo (Malipatil, 2016).

Es la teoría atribucional desarrollada en los años 50 por el psicólogo Fritz Heider, la que proporciona un análisis a cerca de la causalidad a partir de la hipótesis de que las personas tienen la motivación para entender las causas de los eventos que suceden, así como comprender su medio ambiente. Esta teoría permite evaluar cómo las personas perciben su propio comportamiento y el de otros (Alvarez et al., 2015). Desde estos inicios, el enfoque conceptual de la teoría de la atribución se ha incorporado al estudio de prácticamente todos los aspectos de la psicología, desde la clínica hasta la cognitiva, desde el examen de la esquizofrenia hasta la comprensión del esquema; sin embargo, también se ha llevado al ámbito organizacional (Weiner, 2018). En esta teoría se encuentra también razonablemente bien establecido que las percepciones de la responsabilidad individual están influenciadas por variables como la libertad de elección, el control personal, la intencionalidad, la previsión y la capacidad de distinguir el bien del mal (Weiner, 2018). Por lo que, una de las consecuencias inesperadas del crecimiento de la teoría atribucional ha sido debido al incremento en las aplicaciones de dicha teoría a problemas y preocupaciones cotidianas (Weiner, 2014); tales como poca habilidad de comunicación en el salón de clases, el proceso de atribución causal en el deporte y la actividad física, atribución aplicada al estudio del estrés y el afrontamiento, terapias atribucionales, aplicación al conflicto en las relaciones interpersonales e intergrupales, entre otras (Graham y Folkes, 2014). La teoría de la atribución proporciona pues, un marco rico, que incorpora muchos conceptos causales, expectativas de éxito, una variedad de afectos y fenómenos dispares; así como las herramientas para su aplicación (Weiner, 2014). De ahí el interés de los investigadores al relacionar los estilos de atribución con variables como rendimiento académico (Alvarez et al., 2015; Fernández et al., 2015; Gordeeva et al., 2019; Mkumbo y Amani, 2012); motivación y autoeficacia (Becerra-González y Reidl, 2015); influencia en el estilo atribucional en el uso de sustancias y el comportamiento sexual de riesgo (Burnett et al., 2012); interrelaciones entre los enfoques de aprendizaje, su regulación y las estrategias cognitivas (Heikkilä et al., 2011); mejora del estilo atribucional como factor protector en el suicidio (Kleiman et al., 2012) son algunos ejemplos de ello.

En la medición de los estilos atributivos existen un sin número de instrumentos, uno de los más empleados y adaptados es la escala de locus de control de (Rotter, 1966) el cual mide las diferencias individuales en la expectativa generalizada para el control interno-externo con 29 ítems; la Multidimensional-multiattributional Causality Scale (Lefcourt et al., 1979) escala que evalúa el locus de control para la afiliación y el logro con 24 ítems, donde la mitad de los ítems se refieren al éxito y la otra mitad a las experiencias de fracaso; el Attributional Style Questionnaire (Peterson et al., 1982) escala de tres dimensiones asociadas con la descripción de cada evento que califica en las direcciones de mayor internalidad, estabilidad y globalidad, con 18 ítems; el cuestionario de estilos atributivos académicos (AASQ por sus siglas en inglés Academic Attributional Style Questionnaire) (Peterson y Barrett, 1987) consta de 12 eventos académicos hipotéticamente negativos en una escala de tres dimensiones causales de optimismo y pesimismo; el cuestionario de estilos atributivos EALA (Alonso y Sánchez, 1992) este instrumento está conformado por 72 ítems dividido en dos áreas; el área de logros académicos, éste se compone de siete factores: 
externalización e incontrolabilidad de los resultados académicos y fundamentalmente el éxito, atribución del fracaso académico a la falta de esfuerzo, atribución del éxito académico a la habilidad, atribución del fracaso al profesor, atribución del éxito al esfuerzo, externalización e incontrolabilidad del fracaso académico por su atribución a la mala suerte y atribución del fracaso a la falta de habilidad; y el área de relaciones interpersonales, está conformada por cinco dimensiones: internalización del fracaso en las relaciones interpersonales, atribución del éxito en las relaciones interpersonales al esfuerzo, externalización del éxito en las relaciones interpersonales, atribución del éxito en las relaciones interpersonales a la habilidad y externalización del fracaso; adaptación de la Sydney Attribution Scale (Inglés et al., 2008) constituida por 15 ítems en tres factores: atribución del éxito al esfuerzo, atribución del éxito a la habilidad y atribución del éxito a causas externas; en cuanto a las situaciones de fracaso incluyeron 15 ítems distribuidos en tres factores: atribución del fracaso a la habilidad, atribución del fracaso al esfuerzo y atribución del fracaso a causas externas; adaptación del Strategy and Attribution Questionnaire (Heikkilä et al., 2011) este cuestionario consta de 31 ítems con seis dimensiones: preocupación, búsqueda de apoyo social, expectativas de éxito, comportamiento ante las tareas irrelevantes, pensamiento reflexivo, orientación al dominio; y la adaptación a la Escala Atribucional de Motivación al Logro General (Durán-Aponte y Pujol, 2013) para identificar las atribuciones causales sobre el rendimiento general en estudiantes universitarios, consta de 18 ítems con cuatro dimensiones: atribución a la característica de la tarea, atribución al esfuerzo, atribución a la capacidad y atribución a la evaluación de los profesores; entre otros.

Al respecto, existen varios componentes sobre los estilos de atribución los autores van desde la unidimensionalidad del constructo hasta la multidimensionalidad; aunque Mayora-Pernia y Fernández (2015) en su revisión encontraron que el constructo ha evolucionado de ser una variable dicotómica a multidimensional; sin embargo, todos están de acuerdo en incluir el control externo e interno, algunos agregan los factores de estabilidad y su contraparte inestabilidad y la globalidad frente a la especificidad; todas estas dimensiones empleadas para explicar las causas de los éxitos o eventos positivos de los fracasos o los sucesos negativos en la vida. Estableciendo claramente que el locus de control interno se refiere a las atribuciones causales que realizan las personas cuando se consideran capaces de controlar los resultados, gracias a su esfuerzo y capacidades; y el locus de control externo se relaciona con las atribuciones causales que se asignan a la suerte, la acción de otras personas o el control de otros poderes, es decir, la explicación es ajena a la propia persona (Camgoz et al., 2008; O'Donnell et al., 2013; Peterson, 1991). Algunos autores (Aguilar, Gil, Pinto, Quijada y Zúñiga, 2014; Fernández, Arnaiz, Mejía y Barca, 2015; Mayora-Pernia y Fernández, 2015) han realizado investigaciones con estudiantes universitarios, encontrando que el locus de control interno se relaciona significativa y positivamente con el rendimiento académico; mientras que Albert y Dahling (2016) reportaron que cuando el locus de control interno es alto, la relación positiva entre la orientación a la meta de aprendizaje y el autoconcepto académico es fuerte.

En diversos estudios se ha encontrado que el género contribuye de manera significativa en la predicción de los estilos de atribución. Algunas investigaciones han reportado que las mujeres son más propensas a presentar un locus de control interno en comparación con los hombres (Camgoz et al., 2008; Durán-Aponte y Pujol, 2012; Rodríguez-Marín y Inglés, 2011; Suárez-Álvarez et al., 2016; Weiner, 2014). Por el contrario otros autores manifiestan que las mujeres perciben que las consecuencias de sus conductas dependen de factores ajenos a su control, como la suerte, el destino o la participación de otras personas, es decir, muestran un locus de control externo, mientras que los hombres presentan un locus de control interno (Brenlla y Vázquez, 2010; Bulut et al., 2010; Montes-Hidalgo y Tomás-Sábado, 2016; Zaidi y Mohsin, 2013). Sin embargo, hay quienes afirman que no existen diferencias entre el locus de control interno o externo debido al género (Sagone y De Caroli, 2014). Por tanto, el objetivo de este estudio es analizar las diferencias por género a través de los cuatro factores del Cuestionario de Estilos Atributivos en el Área de logros académicos adaptado por Jurado et al. (2016).

\section{METODOLOGÍA}

Se describe quienes fueron los participantes y sus edades, se detalla el instrumento que se utilizó, en este caso un cuestionario, y luego se explica sobre el diseño de la investigación. Se detalla también el procedimiento de la recolección de datos y por último se describe cómo se realizó el análisis de datos.

\section{Participantes}

La muestra de 1285 participantes, 660 (51.4\%) mujeres y 625 (48.6\%) hombres, todos alumnos y alumnas de la Facultad de Ciencias de la Cultura Física de la Universidad Autónoma de Chihuahua. Las edades de las mujeres fluctúan entre los 18 y 26 años, con una media de 20.51 y una desviación estándar de 1.81 años; y las de los hombres fluctúan también entre los 18 y 26 años, pero con una media de 20.83 y una desviación estándar de 1.89 años. La muestra se obtuvo mediante un muestreo por conveniencia, tratando 
de abarcar la representatividad de los diferentes semestres de las licenciaturas que se ofrecen en la la Facultad de Ciencias de la Cultura Física de la Universidad Autónoma de Chihuahua. Instrumento

Cuestionario de Estilos Atributivos en el Área de logros académicos adaptado por Jurado et al. (2016) realizaron tres adaptaciones que fueron: el sentido de los ítems lo giraron hacia el control interno, las opciones de respuesta fueron once e informatizaron el instrumento, este cuestionario consta de 26 ítems tipo Likert que evalúan los patrones atributivos a través de afirmaciones sobre las causas del éxito y el fracaso en distintas situaciones por medio de cuatro factores: Atribución a la suerte con 10 ítems (con ítems como: Por lo general, si he tenido malos resultados en mis calificaciones creo que ha sido por mala suerte, mi mala suerte ha sido muchas veces lo que hizo que no tuviera mejores calificaciones, La suerte ha sido, por lo general, la causa de mis buenas calificaciones en mis estudios, ...), Atribución al esfuerzo con 3 ítems (por ejemplo: Si alguna vez he obtenido bajas calificaciones en mis estudios se ha debido a falta de esfuerzo, Las bajas calificaciones que he obtenido han sido, sobre todo, porque no me he esforzado lo suficiente, Normalmente, si recibo una mala nota en una materia es porque no he estudiado lo suficiente), Atribución a la habilidad con 9 ítems (con afirmaciones como: En mi caso, sacar buenas calificaciones se ha debido a mi propio esfuerzo, Siempre que he obtenido buenas calificaciones ha sido porque he estudiado mucho, Si saco buenas calificaciones es por mi buena capacidad para los estudios...) y Atribución al profesor con 4 ítems (con ítems como: Con frecuencia, mis malas calificaciones se deben a que el profesor no está bien preparado para enseñarme, Si he tenido malas calificaciones en alguna materia a menudo ha sido porque el profesor explicaba mal, A menudo, si he tenido malas calificaciones ha sido porque los profesores no me han explicado bien las lecciones...). Todos los ítems con una de escala de respuesta de 0 a 10 donde 0 es completamente en desacuerdo y 10 completamente de acuerdo (Figura 1).

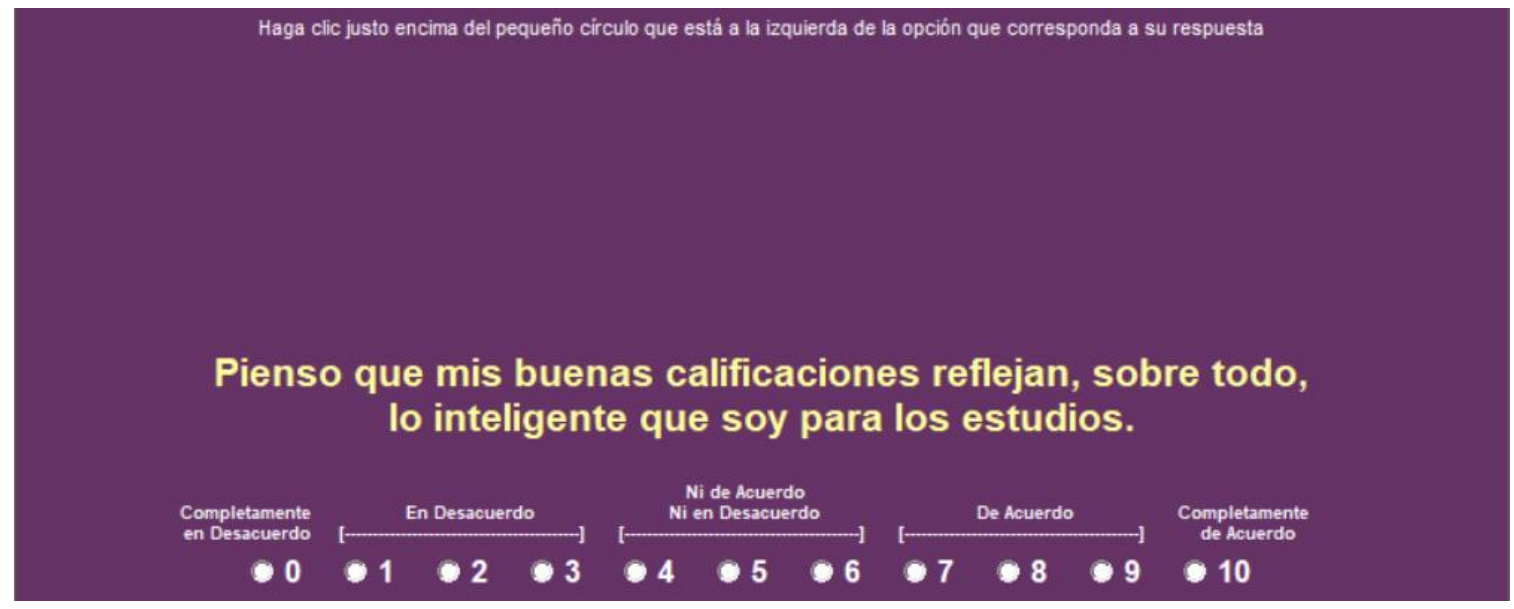

Fig. 1 Ejemplo de respuesta para los ítems del cuestionario

Cabe mencionar que en el presente estudio los ítems de los factores atribución a la suerte (ítems 1, 2, 3, 4, $5,6,7,8,9$ y 10) y atribución al profesor (ítems 23, 24, 25 y 26), se tabularon de tal manera que a mayor puntaje las causas del éxito o fracaso estuvieran referidas a un control externo, ya que por sus nombres se identifican con este tipo de control, mientras que los ítems de los factores atribución al esfuerzo (ítems 11, 12 y 13) y atribución a la habilidad (ítems 14, 1516 y 17) se tabularon de tal manera que a mayor puntaje las causas del éxito o fracaso estuvieran referidas a un control interno (ya que sus nombres hacen referencia a un tipo de control interno). El análisis psicométrico de la escala llevado a cabo por Jurado et al. (2016) indica que el modelo de medición de cuatro factores (atribución a la suerte, atribución al esfuerzo, atribución a la habilidad y atribución al profesor) ajusta de manera aceptable; con índices de consistencia interna iguales o superiores a .70 en todos los factores.

\section{Diseño}

En cuanto al diseño del estudio, se utilizó un enfoque cuantitativo con un diseño descriptivo y transversal tipo encuesta (Hernández et al., 2014). La variable independiente fue género y las variables dependientes las puntuaciones obtenidas en cada uno de los cuatro factores del Cuestionario de Estilos Atributivos en el Área de logros académicos. La Figura 2 muestra la encuesta utilizada para la recolección de datos.

\section{Procedimiento}

Una vez conseguido el permiso de las autoridades educativas correspondientes, se invitó a participar en el estudio a los alumnos y alumnas de las Licenciaturas en Motricidad Humana y Educación Física de la 
Facultad de Ciencias de la Cultura Física de la Universidad Autónoma de Chihuahua. Los que aceptaron participar firmaron la carta de aceptación correspondiente. Luego se aplicó el instrumento por medio de una computadora personal, en una sesión de aproximadamente 30 minutos; en los laboratorios de cómputo de la Facultad de Ciencias de la Cultura Física de la Universidad Autónoma de Chihuahua. Al inicio de cada sesión se hizo una pequeña introducción sobre la importancia de la investigación y de cómo acceder al instrumento. Se les solicitó la máxima sinceridad y se les garantizó la confidencialidad de los datos que se obtuvieran. Las instrucciones de cómo responder se encontraban en las primeras pantallas; antes del primer reactivo del instrumento. Al término de la sesión se les agradeció su participación. Una vez aplicado el instrumento se procedió a recopilar los resultados por medio del módulo generador de resultados del editor de escalas versión 2.0 (Blanco et al., 2013).

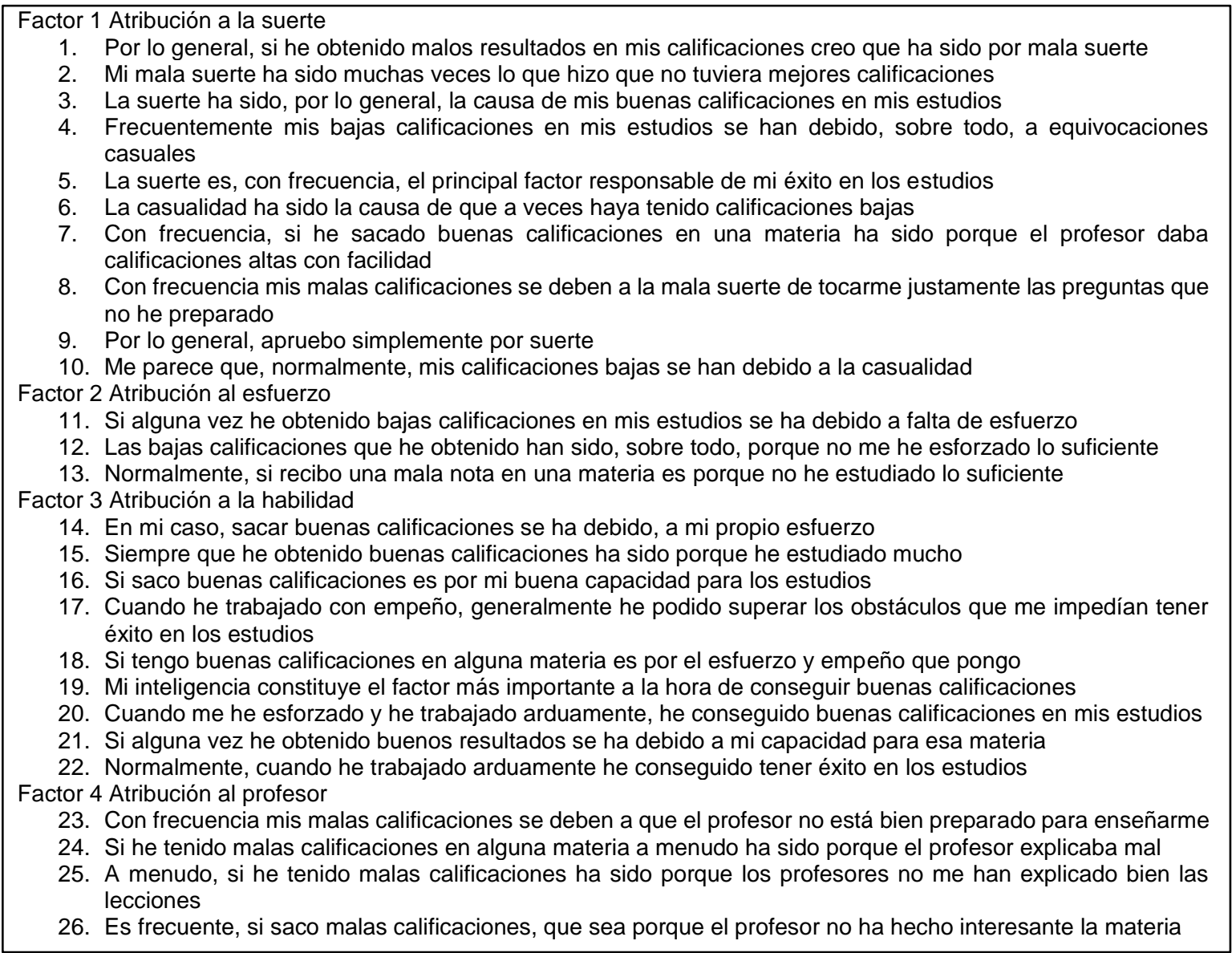

Fig. 2 Encuesta utilizada en este estudio para la recolección de datos.

\section{Análisis de datos}

Se realizaron análisis estadísticos descriptivos (medias y desviaciones estándar) para todas las variables. Posteriormente, después de verificar que los datos cumplían con los supuestos de los análisis estadísticos paramétricos, se llevó a cabo un análisis multivariante de varianza (MANOVA), seguido por los análisis de varianza univariados (ANOVAs), para examinar las diferencias entre mujeres y hombres en cuanto a las puntuaciones obtenidas en cada uno de los cuatro factores del Cuestionario de Estilos Atributivos en el Área de logros académicos. Por otra parte, el tamaño del efecto se estimó mediante el eta cuadrado $\left(\eta^{2}\right)$. Todos los análisis estadísticos se realizaron con el programa SPSS versión 21.0 para Windows. El nivel de significación estadística se estableció en $p=.05$.

\section{RESULTADOS}

La Tabla 1 muestra los valores de las medias (Figura 3) y las desviaciones estándar de las puntuaciones de los cuatro factores estudiados sobre los estilos atributivos en el área de logros académicos, así como los resultados del MANOVA y los subsiguientes ANOVAs. Los resultados del MANOVA mostraron diferencias globales estadísticamente significativas de acuerdo a la variable género en los puntajes promedio de las subescalas de estilos atributivos en el área de logros académicos (Wilks' $\lambda=.937 ; p<.001 ; \eta^{2}=.063$ ). Posteriormente, los resultados de los ANOVAs mostraron que las mujeres reportan mayores niveles de control interno en tres de las dimensiones estudiadas atribución a la suerte $(F=73.239, p<.001)$, atribución 
a la habilidad $(F=12.518, p<.001)$ y atribución al profesor $(F=61.984, p<.001)$. Mientras que en la dimensión atribución al esfuerzo no se encontraron diferencias significativas entre hombres y mujeres.

Tabla 1: Resultados MANOVA y ANOVAs para las diferencias en percepción de burnout académico de acuerdo al género. Los valores descriptivos se presentan como media (desviación estándar).

\begin{tabular}{|l|l|l|l|l|l|}
\hline & $\begin{array}{l}\text { Mujeres } \\
(n=660)\end{array}$ & $\begin{array}{l}\text { Hombres } \\
(n=625)\end{array}$ & $F$ & $P$ & $\eta^{2}$ \\
\hline & & & 21.502 & $<.001$ & .063 \\
\hline Atribución a la suerte & $2.98(0.08)$ & $3.95(0.08)$ & 73.239 & $<.001$ & .054 \\
\hline Atribución al esfuerzo & $7.32(0.07)$ & $7.24(0.07)$ & 0.640 & $>.05$ & .000 \\
\hline Atribución a la habilidad & $7.90(0.05)$ & $7.62(0.06)$ & 12.518 & $<.001$ & .010 \\
\hline Atribución al profesor & $3.95(0.08)$ & $4.86(0.08)$ & 61.984 & $<.001$ & .046 \\
\hline
\end{tabular}

\section{DISCUSIÓN}

Los resultados obtenidos revelan que en la mayoría de los factores de los estilos atributivos en el área de logros académicos, las mujeres en comparación con los hombres se perciben con mayores niveles de control interno, es decir, en comparación con los hombres perciben que sus logros y fracasos académicos están referidos a la dimensión atribución a la habilidad. Resultados que en general concuerda con estudios similares (Durán-Aponte y Pujol, 2012; Rodríguez-Marín y Inglés, 2011); quienes analizaron las atribuciones causales que realizan los estudiantes universitarios en función del género, nivel académico y área de estudio; Además se han realizado estudios donde se reporta que existe evidencia de que el género hace una diferencia en los estilos de atribución, y apoya la afirmación de que las mujeres son más internas que los hombres (Camgoz et al., 2008). Sin embargo, otros autores manifiestan que las mujeres perciben que las consecuencias de sus conductas dependen de factores ajenos a su control, como la suerte, el destino o la participación de otras personas, es decir, muestran un locus de control externo, mientras que los hombres presentan un locus de control interno (Brenlla y Vázquez, 2010; Bulut et al., 2010; Montes-Hidalgo y TomásSábado, 2016; Zaidi y Mohsin, 2013). Por otro lado, existen estudios en los que no se han encontrado diferencias entre el locus de control interno o externo de acuerdo al género (Ahsan, 2017; Sagone y De Caroli, 2014).

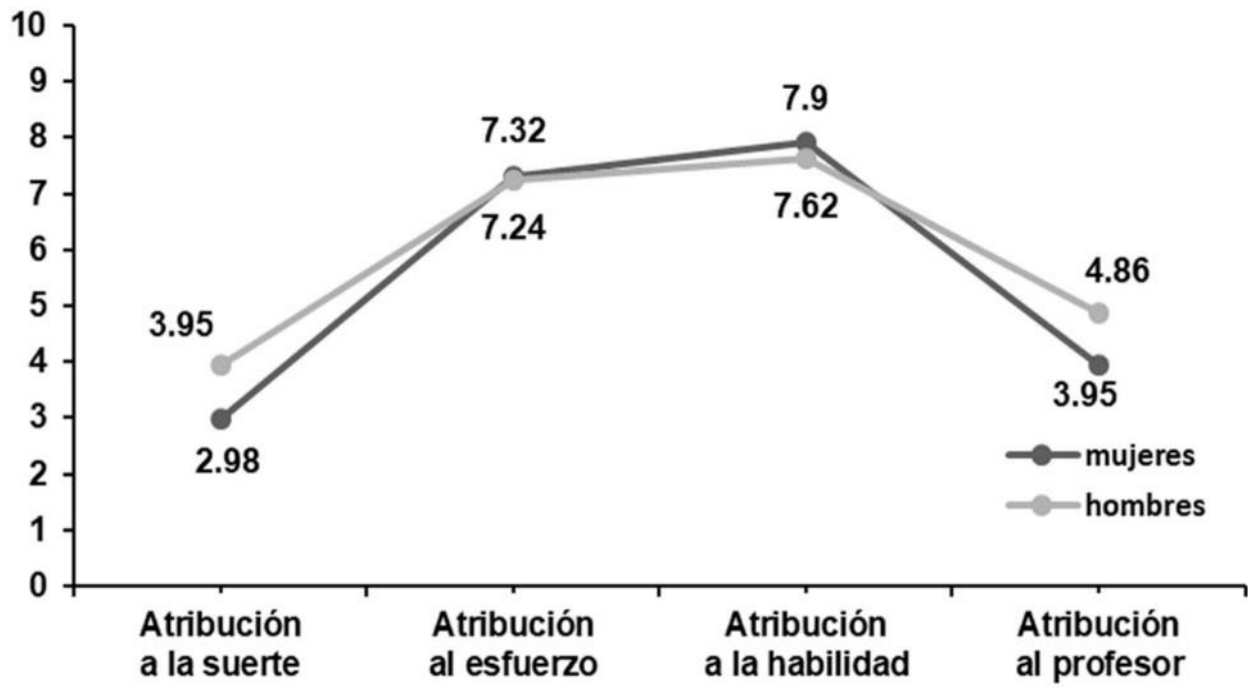

Fig. 3: Perfil de estilos atributivos en el área de logros académicos de acuerdo al género.

Comparar los perfiles de los estilos atributivos en el área de logros académicos sin duda contribuye al mejoramiento de la vida de los estudiantes universitarios, puesto que cuanto más se perciban eficientes académicamente, se juzgarán más positivamente en el presente y en el futuro; y será más probable que tomen control de las circunstancias de la vida cotidiana (Sagone y De Caroli, 2014), lo que justifica llevar a cabo investigaciones que incrementen nuestro conocimiento con respecto a dicha temática. Por ello, el presente estudio intentó determinar las diferencias y similitudes entre hombres y mujeres universitarios 
mexicanos, en cuanto a sus estilos atributivos en el área de logros académicos. Además el presente estudio será de utilidad para la aplicación en diferentes ámbitos como, por ejemplo, estudios de intervención para promover el cambio en los estudiantes hacia un estilo de control más interno.

\section{CONCLUSIONES}

De los resultados mostrados, de su análisis y de su discusión, se pueden obtener las siguientes conclusiones principales:

1) Los resultados del análisis multivariante de varianza, seguido por los análisis de varianza univariados, mostraron que las mujeres en comparación con los hombres reportan mayores niveles de control interno en tres de las dimensiones estudiadas

2) Las mujeres, en mayor medida que los hombres, piensan que sus logros y/o fracasos académicos se deben a causas internas y controlables por el propio sujeto y no a situaciones externas atribuibles a la suerte y a otras personas como los profesores.

3) Las diferencias encontradas entre hombres y mujeres con respecto a sus perfiles de estilos atributivos en el área de logros académicos sugieren que al diseñar cualquier tipo de intervención que tenga como objetivo promover el cambio en los estudiantes hacia un estilo de control más interno habrá que tomar en cuenta a la variable género.

\section{AGRADECIMIENTOS}

A la Secretaría de Educación Pública-Subsecretaría de Educación Superior-Dirección de Superación Académica- Programa para el Desarrollo Profesional Docente (DE-13 -6894) quién financió este estudio.

\section{REFERENCIAS}

Aguilar M. C., Gily O. F. y otros tres autores, Inteligencia Emocional, Estrés, Autoeficacia, Locus de Control y Rendimiento Académico en Universitarios, Enseñanza e Investigación en Psicología, 19(1), 21-35, (2014).

Ahsan A., Attribution Style in Student Athletes, Indian Journal of Health \& Wellbeing, 8(7), (2017).

Albert M. A. y Dahling J. J., Learning Goal Orientation and Locus of Control Interact to Predict Academic Self-Concept and Academic Performance in College Students, https://doi.org/10.1016/j.paid.2016.03.074, Personality and Individual Differences, 97, 245-248, (2016).

Alonso J. y Sánchez J. C., Estilos Atributivos y Motivación: El Cuestionario EAT, En Motivar en la Adolescencia: Teoría, Evaluación e Intervención, pp 81-123 Ediciones de la Universidad Autónoma de Madrid, (1992).

Alvarez G., Bernal Y., Romero G. y Reyes D. R., Atribuciones Causales de los Alumnos del Sua Psicología Acerca de su Rendimiento Escolar Revista Iberoamericana de Producción Académica y Gestión Educativa, (3), (2015).

Becerra-González C. E. y Reidl L. M., Motivación, Autoeficacia, Estilo Atribucional y Rendimiento Escolar de Estudiantes de Bachillerato, Revista Electrónica de Investigación Educativa, 17(3), 79-93, (2015).

Blanco H., Ornelas M. y otros cinco autores, Editor for Creating and Applying Computerise Surveys, doi: 10.1016/j.sbspro.2013.12.105, Procedia Social and Behavioral Sciences, 106, 935-940, (2013).

Brenlla M. E. y Vázquez N., Análisis Psicométrico de la Adaptación Argentina de la Escala de Locus de Control de Rotter, 2, 1-33, 2010.

Bulut N., Serin O. y Şahin S., Factors Affecting the Locus of Control of the University Students, https://doi.org/10.1016/j.sbspro.2010.03.041, Procedia Social and Behavioral Sciences, 2, 449-452, (2010).

Burnett A., Sabato T., Wagner L. y Smith A., The Influence of Attributional Style on Substance Use and Risky Sexual Behavior among College Students, College Student Journal, 48(2), 325-336, (2012).

Camgoz S. M., Tektas O. O. y Metin I., Academic Attributional Style, Self-Efficacy and Gender: A Cross-Cultural Comparison, Social Behavior and Personality: an international journal, 36(1), 97-114, (2008).

Durán-Aponte E. y Pujol L., Diferencias de Género y Área de Estudio en las Atribuciones Causales de Estudiantes Universitarios, Anales de la Universidad Metropolitana, 12(2), 39-51, (2012).

Durán-Aponte E. y Pujol L., Escala Atribucional De Motivación de Logro General (Eaml-G): Adaptación y Análisis de sus Propiedades Psicométricas, Estudios Pedagógicos, 39(1), 83-97, (2013).

Fernández A., Arnaiz P., Mejía R. y Barca A., Atribuciones Causales del Alumnado Universitario de República Dominicana con Alto y Bajo Rendimiento Académico, https://doi.org/10.17979/reipe.2015.2.1.1319 Revista de Estudios e Investigación en Psicología y Educación, 2(1), 19-29, (2015). 
Gordeeva T., Sheldon K. y Sychev O., Linking Academic Performance to Optimistic Attributional Style: Attributions Following Positive Events Matter Most, https://doi.org/10.1007/s10212-019-00414-y, European Journal of Psychology of Education, 1-28, (2019).

Graham S. y Folkes V. S., Attribution Theory: Applications to Achievement, Mental Health, and Interpersonal Conflict, Taylor \& Francis, (2014).

Heikkilä A., Niemivirta M., Nieminen J. y Lonka K., Interrelations among University Students' Approaches to Learning, Regulation of Learning, and Cognitive and Attributional Strategies: A Person Oriented Approach, Higher Education, 61(5), 513-529, (2011).

Hernández R., Fernández C.y Baptista P., Metodología de la Investigación, McGraw- Hill, México, (2014).

Inglés C. J., Rodríguez-Marín J. y González-Pienda J. A., Adaptación de la Sydney Attribution Scale en Población Universitaria Española, Psicothema, 20(1), 166-173, (2008).

Jurado P. J., Blanco H., Zueck M. C. y Peinado J. E., Composición Factorial del Cuestionario de Estilos Atributivos Área de Logros Académicos En Universitarios Mexicanos, https://doi.org/10.4067/S0718-50062016000600008, Formación Universitaria, 9(6), 83-92, (2016).

Kleiman E. M., Miller A. B. y Riskind J. H., Enhancing Attributional Style as a Protective Factor in Suicide, https://doi.org/10.1016/j.jad.2012.05.014, Journal of Affective Disorders, 143, 236-240, (2012).

Lefcourt H. M., Von Baeyer C. L., Ware E. E. y Cox D. J., The Multidimensional-Multiattributional Causality Scale: The Development of a Goal Specific Locus of Control Scale, Canadian Journal of Behavioural Science/Revue canadienne des sciences du comportement, 11(4), 286, (1979).

Malipatil R. P., Terminology and Theoretical Framework of the Study, En Socio-Psychological Correlations of Aggressive Behaviour, Locus of Control, Emotional Intelligance and Will to Win of the Sportsman, pp 10-37 Lulu Publication, (2016).

Mayora-Pernia C. A. y Fernández N., Locus de Control y Rendimiento Académico en Educación Universitaria: Una Revisión Bibliográfica, doi: 10.15359/ree.19-3.16, Revista Electrónica Educare, 19(3), 1-23, (2015).

Mkumbo K. A. K. y Amani J., Perceived University Students' Attributions of Their Academic Success and Failure, https://doi.org/10.5539/ass.v8n7p247, Asian Social Science, 8(7), 247-255, (2012).

Montes-Hidalgo J. y Tomás-Sábado J., Autoestima, Resiliencia, Locus de Control y Riesgo Suicida en Estudiantes de Enfermería, https://doi.org/10.1016/j.enfcli.2016.03.002, Enfermería Clínica, 26(5), (2016).

O'Donnell S., Chang K. y Miller K., Relations among Autonomy, Attribution Style, and Happiness in College Students, College Student Journal, 47(1), 228-234, (2013).

Peterson C., The Meaning and Measurement of Explanatory Style, https://doi.org/10.1207/s15327965pli0201_1, Psychological Inquiry, 2(1), 1-10, (1991).

Peterson C. y Barrett L. C., Explanatory Style and Academic Performance among University Freshman, Journal of Personality and Social Psychology, 53(3), 603-607, (1987).

Peterson C., Semmel A. y otros cuatro autores, The Attributional Style Questionnaire, Cognitive therapy and research, 6(3), 287-299, (1982).

Rodríguez-Marín J. y Inglés C. J., Diferencias de Sexo y Curso en Autoatribuciones Académicas de Estudiantes Universitarios, Revista Mexicana de Psicología, 28(2), 173-181, (2011).

Rotter J. B., Generalized Expectancies for Internal Versus External Control of Reinforcement, Psychological Monographs: General and Applied, 80(1), 1-28, (1966).

Sagone E. y De Caroli M. E., Locus of Control and Academic Self-Efficafy in Universitary Students: The Effects of SelfConcepts, https://doi.org/10.1016/j.sbspro.2013.12.689, Social and Behavioral Sciences, 114, 222-228, (2014).

Salvador C. y Mayoral L., Influencia de los Estilos de Atribución en la Inteligencia Emocional de los Estudiantes Argentinos, Revista Electrónica Interuniversitaria de Formación del Profesorado, 14(1), 243-251, (2011).

Suárez-Álvarez J., Pedrosa I., García-Cueto E. y Muñiz J., Locus of Control Revisited: Development of a New BiDimensional Measure, https://doi.org/10.6018/analesps.32.2.200781, Anales de psicología, 32(2), 578-586, (2016).

Tavakolizadeh J. y Ebrahimi S., El Efecto de Enseñar Estrategias de Aprendizaje Autorregulado sobre Estilos de Atribución de los Alumnos, Electronic Journal of Research in Educational Psychology, 9(25), 1087-1101, (2011).

Visdómine-Lozano J. C. y Luciano C., Locus de Control y Autorregulación Conductual: Revisiones Conceptual y Experimental, International Journal of Clinical and Health Psychology, 6(3), 729-751, (2006).

Weiner B., Searching for the Roots of Applied Attribution Theory, En Attribution Theory: Applications to Achievement, Mental Health, and Interpersonal Conflict, por S. Graham, Folkes V. S., pp 1-16 Taylor \& Francis, (2014).

Weiner B., Introduction: The Role and Function of Attribution Theory in the Organizational Sciences, En Attribution Theory: An Organizational Perspective, por M. J. Martinko, Taylor \& Francis, (2018).

Zaidi I. H. y Mohsin M. N., Locus of Control in Graduation Students, International Journal of Psychological Research, 6(1), 15-20, (2013). 